\title{
21 IMPLICATIONS OF THE THEORY OF AUTOPOIESIS FOR THE DISCIPLINE AND PRACTICE OF INFORMATION SYSTEMS
}

\author{
Ian Beeson \\ Faculty of Computer Studies \& Mathematics \\ University of the West of England \\ Bristol \\ England
}

\begin{abstract}
The theory of autopoiesis, developed in biology by Maturana and Varela, deprives information of any significant role in self-producing systems, because such systems are organizationally closed. After exploring the rejection of the notion of information, and laying out the main tenets of the theory, this paper considers some of the implications of the theoretical position for the discipline and practice of information systems (IS). The chief consequence is to shift focus from abstraction, representation, and design toward cooperation and use. The paper goes on to discuss different approaches to applying the theory of autopoiesis in IS. Some benefit might, for instance, be had from using the ideas as metaphors. The role of information is seen to be restored in Luhmann's development of an autopoietic theory for social systems. A more radical use of the theory in IS would be to develop the basic ontology proposed by Maturana and Varela. A start on this is made from a phenomenological perspective.
\end{abstract}

\section{NO INFORMATION, NO REPRESENTATION}

In the theory of autopoiesis put forward by Maturana and Varela (1980), information is not considered relevant to a system's self-production. In their early work on autopoiesis and cognition, they write: 
Notions such as coding and transmission of information do not enter in the realization of a concrete autopoietic system because they do not refer to actual processes in it. The notion of coding is a cognitive notion which represents the interactions of the observer, not a phenomenon in the observed domain. The same applies to the notion of regulation (Maturana and Varela 1980, p. 90).

And later in the same work:

Notions such as coding, message or information are not applicable to the phenomenon of self-reproduction; their use in the description of this phenomenon constitutes an attempt to represent it in the language of heteropoetic design (Maturana and Varela 1980, p. 102).

Beer (1980), in the preface to Maturana and Varela's book, expresses alarm and then conversion:
All of this is totally alien to what we (most of us working in cybernetics) have believed. Information, including codes and messages and mappings, was indeed for us the whole story of the viable system. If one thinks of reproduction, for example, as the process of passing on a DNA code from an aging set of tissues to an embryonic set of tissues, then the survival of the code itself is what matters....The whole outlook turns out to be wrong....I do not know whether the authors' arguments about information led me to understand their concept of autopoiesis, or vice versa. What I am now sure about is that they are right. Nature is not about codes; we observers invent the codes in order to codify what nature is about (p. 69).

In a later work in which they present their ideas to a general audience, Maturana and Varela (1987) reject the idea that the organism, via its nervous system, builds an internal representation of the world outside:

The most popular and current view of the nervous system considers it an instrument whereby the organism gets information from the environment which it then uses to build a representation of the world that is uses to compute behavior adequate for its survival in the world....We know, however, that the nervous system as part of an organism operates with struc- 
tural determination. Therefore, the structure of the environment cannot specify its changes, but can only trigger them. We as observers have access both to the nervous system and to the structure of the environment. We can thus describe the behavior of an organism as though it arose from the operation of its nervous system with representations of the environment or as an expression of some goal-oriented process. These descriptions, however, do not reflect the operation of the nervous system itself. They are good only for the purpose of communication among ourselves as observers. They are inadequate for a scientific explanation (Maturana and Varela 1987, p. 129).

They reject the notion of the nervous system or brain as an information processing device:

In each interaction, it is the nervous system's structural state that specifies what perturbations are possible and what changes trigger them. It would therefore be a mistake to define the nervous system as having inputs or outputs in the traditional sense....What is necessary... is to recognize the nervous system as a unity defined by its internal relations in which interactions come into play only by modulating its structural dynamics, i.e., as a unity with operational closure. In other words, the nervous system does not "pick up information" from the environment, as we often hear. On the contrary, it brings forth a world by specifying what patterns of the environment are perturbations and what changes trigger them in the organism. The popular metaphor of calling the brain an "information-processing device" is not only ambiguous but patently wrong (Maturana and Varela 1987, p. 169).

And they reject the idea of information being transmitted in communication:

Our discussion has led us to conclude that there is no "transmitted information" in communication. Communication takes place each time there is behavioral coordination in a realm of structural coupling....According to [the] metaphor of the tube, communication is something generated at a certain point. It is carried by a conduit (or tube) and is delivered to the receiver at the other end. Hence, there is a something that is communicated....We usually speak of the "information" contained in a 
picture, an object, or, more evidently, the printed word.... According to our analysis, this metaphor is basically false. It presupposes a unity that is not determined structurally, where interactions are instructive, as though what happens to a system in an interaction is determined by the perturbing agent and not by its structural dynamics. It is evident, however, even in daily life, that such is not the case with communication: each person says what he says or hears what he hears according to his own structural determination; saying does not ensure listening....The phenomenon of communication depends on not what is transmitted, but on what happens to the person who receives it. And this is a very different matter from "transmitting information" (Maturana and Varela 1987, p. 196).

It seems from these excerpts that the theory of autopoiesis raises a serious challenge to the discipline and practice of information systems, at least in so far as these are built on some fundamental concept of information. What are the implications for information systems if the theory of autopoiesis does in fact provide an accurate account of the operation of living systems? If information is deprived of its central place in information systems, what remains of the discipline and practice? Could information systems be beneficially reconceptualized as autopoietic systems themselves? If we accept that human beings are autopoietic systems, what will be the nature of their interactions with-or in-information systems?

Despite the apparent importance and possibly serious implications of the theory of autopoiesis for information systems (IS), there has been little discussion of autopoiesis in the IS literature. Winograd and Flores (1986) and Mingers (1991, 1995) provide notable exceptions, but in general, autopoiesis has made little theoretical or practical impact in the IS field. Indeed, it has been taken up with rather more enthusiasm in a number of other fields (see Mingers 1995), such as law, family therapy (Dell 1985), sociology (Luhmann 1989, 1995), management (von Krogh and Roos 1996), and public administration (Kickert 1993). Capra (1997) gives a general treatment (and provides the title for this section [p. 263]).

The reluctance to engage with the theory of autopoiesis in IS may be due to a general under-theorization in our field. Or it may be that the attack from autopoiesis is so central that IS cannot deal with it without a risk of disintegration.

At any event, the remainder of this paper, after first giving a summary account of the main tenets of autopoiesis theory, will attempt to draw out some possible implications and applications of it in the IS field. 


\section{THE THEORY OF AUTOPOIESIS}

Maturana and Varela's theory of autopoiesis identifies living systems with autopoietic (self producing) systems. The theory gives a comprehensive and economic account of living beings from the simplest unicellular organisms to the most complex multicellular organisms, and extends beyond organisms to social systems.

Living beings are defined as autonomous unities with autopoietic organization. Such a unity is distinguishable (by an observer) against a background. An autopoietic system exists as a network of relations and processes which continuously produce the components which realize that network as a concrete unity. Included among the components which a living system specifies and produces for itself is the system's own boundary.

A key distinction is made between organization and structure: the organization of a system is defined as the relations that define it as a unity (of a certain class) and determine the dynamics of interaction and transformations which it may undergo as that unity; while its structure is defined as "the components and relations that actually constitute a particular unity and make its organization real" (Maturana and Varela 1987, p. 47). The same organization may be realized in different structures. Structures are not static, but constantly changing as the unity interacts with its medium (environment).

Autopoietic systems are organizationally (or operationally) closed. That is to say, the behavior of the system is not specified or controlled by its environment but entirely by its own structure, which specifies how the system will behave under all circumstances. It is as a consequence of this closure that living systems cannot have "inputs" or "outputs"-nor can they receive or produce information - in any sense in which these would have independent, objective reality outside the system. Put in another way, since the system determines its own behavior, there can be no "instructive interactions" by means of which something outside the system determines its behavior. A system's responses are always determined by its structure, although they may be triggered by an environmental event. To an observer who can see both the system and its environment, it might seem as if the system's behavior is controlled by the environment, but this is a feature of the observation, not of the operational reality. Autopoietic systems are thus structure-determined. From the organizational closure of living systems follows their autonomy and their individuality: each individual has its own autonomous ontogeny (its own separate development and history), which is neither controlled by its environment, nor subsumed into its class or species.

Although organizationally closed, a system is not disconnected from its environment, but in fact in constant interaction with it. Maturana and Varela 
(1987) call this ongoing process "structural coupling" (p. 75). System and environment (which will include other systems) act as mutual sources of perturbation for one another, triggering changes of state in one another. Over time, provided there are no destructive interactions between the system and the medium in which it realizes itself (i.e., its environment), the system will appear to an observer to adapt to its environment. What is in fact happening, though, is a process of structural "drift" occurring as the system responds to successive perturbations in the environment according to its structure at each moment.

The significant shift in thinking here is that the changes in a living being which result from its interaction with its environment are seen as being brought about (triggered) by a disturbing agent in the environment, but not determined by it. It is rather the structure of the disturbed system (the living being) that determines the changes. The environment provides perturbations for the living being (and vice versa) but not instructions. To say it again, this is what gives each unity its autonomy. For human beings, this is where free will lies.

The ideas of structural coupling and structure determined systems give us a new explanation for phenomena we have previously explained by concepts such as representation and adaptation. Winograd and Flores (1986) give a good example:

The frog with optic fibers responding to small moving dark spots does not have a representation of flies. As a result of structural coupling, the structure of the nervous system generates patterns of activity that are triggered by specific perturbations and that contribute to the continued autopoiesis of the frog. Of course, the changes of structure that led to the frog's nervous system would not have been supportive of autopoiesis if the frog had to compete for food in a flyless environment. But it is an error to assume that the structure reflects a knowledge of the existence of flies (p. 46).

The nervous system of the frog, and of other animals, is operationally closed: it is organized as "a network of active components in which every change of relations of activity leads to further changes of relations of activity" (Maturana and Varela 1987, p. 164). Nevertheless because the nervous system couples an organism's sensory and motor surfaces through a complex and dynamic web of neurons, it greatly increases the versatility and plasticity of the organism, and expands its range of possible states and of observable behaviors. This in turn opens up new dimensions of structural coupling for the organism (Maturana and Varela 1987, p. 175).

Recurrent interactions among organisms with a nervous system will give rise to what Maturana and Varela (1987) call "third-order" couplings and unities 
(where cells are first-order, and multicellular organisms second-order, unities). Couplings between organisms are likely to arise as the natural result of the congruence between their respective ontogenic drifts (Maturana and Varela 1987, p. 181). A third-order unity - a "social system" of animals or peopleitself displays autopoietic organization to the extent that the network of participating individual organisms co-produce it through their reciprocal structural coupling (Maturana and Varela 1987, p. 193).

The reciprocal coordination mutually triggered among the members of a social unity Maturana and Varela $(1987$, p. 193) call communication. So defined, communication is an aspect of social behavior, emerging out of structural coupling and drift in social groups, and not a separate or distinct mechanism suddenly appearing in evolutionary development.

Communication is then the basic behavior from which language (or "languaging") emerges. Human linguistic behavior is a domain of reciprocal ontogenic structural coupling which we human beings establish and maintain together. Although an observer is able to describe words as designators of objects or situations in the world, the operational reality of our use of language with one another reflects a structural coupling in which words are "ontogenically established coordinations of behavior" (Maturana and Varela 1987, p. 208).

Once we are able to use language, we can describe ourselves and our circumstances. In fact, we become able to describe the world unlimitedly. We are also able to become an observer of living systems in their environments. None of this, however, releases us from the reality of being structure-determined systems ourselves.

Just as Maturana and Varela put communication before language, making communicative behavior, arising from structural coupling, a precursor of linguistic behavior, so they suggest that mind and consciousness, far from being prior to language, arise out of it. As they put it, "language is a condition sine qua non for the experience of what we call mind" (1987, p. 231). They suggest further that the idea of self may arise out of the use of names and that the idea of "I" similarly arises first as a linguistic distinction, which locates us as individuals in a network of linguistic interactions.

The theory of autopoiesis is a remarkable edifice. It was built originally on experimental research in perception, which showed that the nervous system was best understood and explained as generating phenomena (e.g., color perception) on the basis of complex activity among neurons, rather than as filtering or mapping external stimuli. It has been expanded to provide a full explanation of living systems as autopoietic systems which are structure-determined and structurally coupled to their environment (including to one another). It reaches from single-celled organisms to social systems.

In the construction of this theory, Maturana and Varela have inverted and challenged previous theories. They give the individual primacy over the species, 
and relegate reproduction to a secondary function of the living system. Assertion of the autonomy of the individual has political implications. They solve the problem of the chicken and the egg by stating unequivocally that the chicken came first. They deny that genes contain the information that specifies a living thing.

They turn the Cartesian cogito ergo sum entirely upside down. Sum ergo cogito would be closer to their position: being precedes thinking. Indeed, thinking, in the sense of conscious thought, comes at the end of a chain which starts with autopoiesis and proceeds through structural coupling, communication, and language before it reaches thinking. Alternatively, the theory can be read as generalizing thinking (as cognition) so that it becomes a property of all living systems. As Maturana and Varela say at the beginning of The Tree of Knowledge, "We will propose a way of seeing cognition not as a representation of the world 'out there,' but rather as an ongoing bringing forth of a world through the process of living itself' $(1987$, p. 9).

On either interpretation of thinking, the theory of autopoiesis puts being before it, and so, unusually, develops an approach to cognition that is existential and ontological rather than epistemological. Let us turn our attention now to some of the implications of this theory for the discipline and practice of information systems.

\section{IMPLICATIONS FOR INFORMATION SYSTEMS}

The rejection of the notions of externally present information, of the transmission of information in communication, of the representation of the outside world, and of instructive interactions, all appear to undermine a discipline and practice of information systems which is grounded in precisely these ideas and possibilities. If there is no information objectively present in the world, how can we as IS practitioners or theoreticians abstract it and make a representation of it as a basis for IS design? If we do not have a representation of the outside world in our heads, what is it in the user's mind that analysts are seeking to elicit as a requirement, or in the analyst's mind when a new system is invented and modeled? If information is not transmitted in communication, what is the basis for the design and use of systems of human-computer interaction, or even of systems of human to human interaction, or indeed of information systems in general? If there are no instructive interactions, how can managers control a workforce, or how can the implementation of a design produce desired user behavior?

All of these issues need to be rethought if autopoiesis is taken seriously. To begin with, we need to rethink the idea and position of information in an information system. The notion that information (likewise knowledge) is some sort of stuff that can be unproblematically detached from people and stored in 
a machine or sent across a network has to be challenged. Information must be seen instead as a feature of behavioral coordination.

Similarly, we have to be prepared to jettison the notion that organizational or social reality can be represented in its true essence in a diagram or formal description. At best, such descriptions can provide the basis for the coordination of activity among autonomous individuals. If no representation of an information system can be made that will be compelling for any human being involved in its construction or use, no final description or specification of an information system can be made that will guarantee or fix its implementation. The emphasis in system design methodologies on formal specification must be subordinated to the fostering of mutual understanding and coordinated action in the development team (in which formal descriptions can certainly play a part, but not a determining one). A fully automated system might be specifiable, but not an information system which contains human users as well as machines.

The jettisoning of core systems concepts such as control, order, and hierarchy seems shocking, yet this also follows from the impossibility of objective representations and instructive interactions. The presence of order, control, and hierarchy in a system, according to Maturana and Varela, is the construction of an observer, describing a sequence of actions from an external perspective; the effective operational reality, as ever, is one of structure-determined interaction, coupling, and drift. To put it bluntly, managers and designers cannot, finally, determine what other people do. They cannot achieve just what they want, either with other people or with machines which have human users.

As has been suggested elsewhere (Beeson and Davis 2000), systems theory could be usefully reformulated by bringing in ideas from complexity theory. Systems theory would benefit by being shifted away from its emphasis on control and regulation and toward an emphasis on emergence. Emergence can be seen as fundamentally uncontrollable, and as arising out of the interactions and accomplishments of the individuals participating in the system. Such a shift of emphasis seems to be compatible with an autopoietic perspective.

The sharp separation made in autopoiesis theory between living and nonliving systems carries with it the implication for information systems that human beings cannot be straightforwardly replaced by machines in a program of automation. Automation, of course, remains possible, but the substitution of machines for people creates a new reality, not a simple continuation of the old one.

The strong program in artificial intelligence, which seeks to build machines that are intelligent in the same way that humans are intelligent, cannot proceed, if autopoiesis theory is correct, by building internal representations of the external world in the cognitivist tradition. Similar obstacles lie in the path of the designers of decision support systems, to the extent that they also proceed on the supposition that users are working with an internal representation of the world which can be supported, reflected, and complemented by the system's representation of the world. Weaker programs, which make no claims about how 
people's minds work but simply try to produce intelligent machine behavior by whatever method that works, can continue, but then there will be no obvious justification for using a cognitivist approach and so questions will arise about which methods to pursue.

In the area of human-computer interaction, the theory of autopoiesis challenges notions of representation (e.g., the system's model of the user and the user's model of the system) and of the transmission of information in such interaction. Winograd and Flores (1986) respond to these problems (provoked for them not only by the theory of autopoiesis but also by consideration of Heideggerian phenomenology) by shifting the focus from human-computer interaction toward computer enabled or supported human-human interaction. They move in particular toward a language action perspective and see possibilities for computer systems that support the conduct of conversations. This, and the development of $\mathrm{HCI}$ in the direction of computer supported cooperative work (CSCW), seems generally compatible with an autopoietic perspective, insofar as the emphasis shifts away from models and messages and towards coordination of behavior.

Broadening the debate from HCI and CSCW toward the general question of the use of computer systems, an autopoietic analysis will focus on how structural coupling occurs between a human user and a computer system. The user is an autopoietic system but the computer system, although it is a structuredetermined system, is not an autopoietic one (since it is not self-producing in the manner of a living system). Therefore, all the drift in the process of coupling will come from the user side. The user will do all the adapting, and here is the source both of user resentment and of invention and surprise in the use of computers.

When we put together the impossibility of final descriptions and the impossibility of instructive interactions, it becomes clear why configuring the user (Grint and Woolgar 1997; McLoughlin 1999) is so difficult in IS practice. We can see that establishing the users' requirement(s) in any final sense is not possible (which throws the project of requirements engineering into disarray). We can also see that constructing the system that the users really wanted is not possible either-even if you build participation and prototyping into the heart of your development methdodology-because the structural plasticity of both the users and the technology create an open horizon of actual use which cannot be prefigured in design, even in principle.

The burden of the argument in this section has been that, if information systems discipline and practice are to respond to the challenges posed by the theory of autopoiesis, attention will have to shift away from design and abstraction and toward implementation and use. It will be in the emergent structural coupling of user and computer system, and of users working through the computer system, that the information system will be realized, and this cannot be determined either in advance or in operation by its design or specification, nor by management. 
In the next section, some alternative ways of developing the ideas from autopoiesis theory in the field of information systems will be considered.

\section{DEVELOPING THE THEORY OF AUTOPOIESIS IN IS}

Briefly, this section discusses the use of autopoiesis as metaphor, the development by Luhmann of an autopoietic approach to social systems, and further development for IS of the ontology proposed in the theory of autopoiesis.

\subsection{Autopoiesis as Metaphor}

Among the many metaphors or images of organization proposed by Morgan (1997, Chapter 8), autopoiesis is discussed under the broader general metaphor of organization as flux and transformation. Morgan notes three principal features of Maturana and Varela's theory of living systems, namely autonomy, circularity, and self-reference. He traces the consequences for organizations of treating interactions with the environment as internally determined. He suggests we can see organizations as always attempting to turn their environments into extensions of their own identity, which implies organizational focus on establishing and projecting an identity and on shaping relations between the organization and its environment in the organization's interest. He expresses concern himself that such emphases will tend to produce narcissistic, egocentric organizations that are over-concerned with themselves and with reducing the environment to something to be absorbed.

Morgan's reading of the theory of autopoiesis may well be one which makes sense to organizational members, but it also seems to be a misreading, in that the notion of structural coupling has been transmuted into one of extension or takeover. The self-producing system cannot instruct or control its environment in structural coupling, only perturb it. The system can certainly interact destructively with its environment, but that would break the coupling.

The danger of taking such ideas as autopoiesis metaphorically is that they may be taken on in a cosmetic or superficial fashion. They may well be taken on by powerful people in organizations as a way of strengthening their own position. Autopoiesis is probably a good example of a theory that could be used by managers and consultants as a new form of obfuscation and as a justification for further extensions of management power.

It certainly is the case, on the other hand, that using powerful ideas metaphorically can stimulate new understanding and open up new possibilities of action. Kickert (1993), for example, after reviewing the relevance of the theory of autopoiesis in the field of public administration, concludes: 
The possibilities of a strict conversion of the autopoiesis model into a valid model that can be used in the administrative sciences are limited. The usefulness of the model does not seem to lie in strict adherence to the original and literal translation, but rather in its power as a source of creative lateral thinking (p. 276).

We may in information systems be able to use the ideas of autopoiesis metaphorically to deepen our understanding of the tendency of IS-construed here as an assembly of interested parties using and maintaining a technical system - to reproduce themselves in a manner that is conservative and hard to change. We may use the ideas to see or explain why users' or designers' behavior seems to be stuck sometimes in persistent patterns or repertoires. The difficulties of system integration or of user-developer participation may be better understood from a perspective of structural coupling than from one of rational design or negotiation. More positively, autopoiesis may provide an explanation of why users and systems can be resilient sometimes in adverse circumstances. We can call on ideas from autopoiesis to support actions in favor of individual autonomy or of a gradualist approach to change.

When it comes to discussing information systems, it may be that a metaphorical use of the theory of autopoiesis is the only use realistically open. If we treat information systems as social or sociotechnical systems, we are seeing them as third order unities. However, whether a third-order unity is itself an autopoietic system is a moot point. The generalization of Maturana and Varela's theory from biological to social systems remains problematic (for them as well as others). An observer may see a third order unity as autopoietic on the grounds that its realization depends on the autopoiesis of the (second-order) unities which integrate it. But it could be argued that unless the third-order system is itself defined by relations between components which in turn produce the relations, it is not truly an autopoietic system. To see an information system as a third-order unity, we would have to understand it as in some sense producing and maintaining its own components (which include human beings) and the relations between them. This question remains open.

\subsection{Autopoietic Social Systems}

Luhmann (1995) develops an ambitious answer to this question by proposing an autopoietic theory of social systems. Luhmann takes the basic ideas of autopoiesis to present social systems as continuous self-referential productions. In what Knodt (1995, p. xxiii) calls "a brilliant move," Luhmann drops the premise that social systems are living systems, thereby severing the autopoiesis of social systems from their biological foundations. Faced with the question of how social systems can be said to produce the people that compose 
them, Luhmann's answer is that the basic elements of social systems are not people but communications.

Social systems in this formulation are seen as being recursively produced by communications. Communication is no longer seen, as in Maturana and Varela, as an aspect (only) of behavioral coordination, but now occupies center stage. The central focus on living or being is replaced by a central focus on meaning and its achievement. Meaning is conceived in terms of an interpenetration of psychic and social systems. Although he describes consciousness and the formation of social systems as evolutionarily co-emergent, Luhmann separates psychic from social systems, so establishing the autonomy of the latter. He thereby rejects the idea of the social as derived from a realm of intersubjectivity, and the idea of communication as an interaction between subjects or a transmission between consciousness, concentrating instead on the social system as an autonomous (organizationally closed) system of communications.

Luhmann's work entails a comprehensive refoundation of sociology, and this brief sketch does not do justice to it. It should, however, be clear that, in developing the theory of autopoiesis for application to social systems, Luhmann has produced a decisive break from the original biological theory of Maturana and Varela. Autopoiesis in his hands takes on the character of an abstract philosophy, leaning in an epistemological rather than an ontological direction.

Luhmann's development of the theory of autopoiesis looks better aligned with mainstream thinking in IS than does the biological theory. Luhmann's deletion of the subject and rejection of intersubjectivity as the basis of communication sits well with IS perspectives, which have always been more interested in information flows and structures than in individual consciousness or cooperative action. His analysis of communication as a synthesis of information, utterance, and understanding appears to restore information to a central place in theory, and has at least some echoes of the language action approach developed (e.g., by Winograd and Flores 1986) from the work of Austin and Searle. Luhmann also has a place for codes and coding in his theory ("Coded events operate as information in the communication process, uncoded ones as disturbance (noise)" [1995, p. 142]).

Radical as it is in its rewriting of sociology and of autopoiesis, Luhmann's theory looks more familiar and more accessible in IS terms than Maturana and Varela's precursor. After all, it is an abstract systems theory! Luhmann's work should be a fruitful source from which to develop autopoietic perspectives within IS. One obvious point of departure would be to use Luhmann's analysis in a critique of the language action approach in IS (already usefully criticized from a Habermasian perspective in, e.g., Dietz and Widdershoven 1991).

The language action approach, at least as formulated by Winograd and Flores, is compatible with an autopoietic perspective. However, even in their hands, the focus of interest shifts away from the people endeavoring to communicate toward the system of exchange of messages. This is certainly compatible with a Luhmannian view of an information system as a social system 
constituted by communications, but is somewhat removed from Maturana and Varela's original conception of third-order unities as living systems.

\subsection{Taking the Ontological Road}

Dell (1985), in his illuminating comparison of the work of Maturana and Bateson, remarks that ontology is "the road not taken" in Bateson's thinking and suggests that the biological ontology delineated in Maturana's work could provide a sound foundation for the social and behavioral sciences.

What would an ontological development of the theory of autopoiesis be like in IS? Ontology is the branch of philosophy concerned with what things exist in the world rather than with how we know things (the domain of epistemology). The discipline of IS, no doubt due to its own history, has always been more oriented toward epistemology than ontology. It has not been so interested in the nature of things and persons in the world, as in the qualities of the entities, relationships, and procedures in its representations, and how they may be established as a basis for a design. This is why Luhmann's version of autopoiesis looks on the face of it easier to live with than Maturana and Varela's.

However, it might be possible to develop a richer ontological vein in IS by starting from a base in the theory of autopoiesis. Winograd and Flores had the same impulse ("The most important designing is ontological" [1986, p. 46]), but their main influence has probably been through the language action approach, which has a mainly epistemological thrust (toward the coordination of understandings and commitments).

One way to develop an ontological perspective is to return to the phenomenology of the individual, or subject, abandoned by Luhmann. (To Luhmann's question, "Which one of the five billion?"-which he uses to "explode the fiction of the transcendental subject" [Knodt 1995, p. xxvi], we could answer "Any.") Maturana and Varela provide us with a basic ontology of a world populated by structure-determined unities, and the theory of autopoiesis affirms the primacy of the individual over the class. Some organisms, we also know, have the capacity to be observers.

When an autopoietic system interacts with its medium, structural changes may be triggered in it. This is the way system and medium become structurally coupled. We expect the ontogenies of coupled individuals in the same class of unities to be similar by virtue of their couplings and their existence within a common medium. A shared ontology might, therefore, be expected to emerge among human beings who are part of the same sociocultural system (as well as belonging to one species).

The exploration and articulation of such shared worlds has already been undertaken by existential phenomenologists, particularly Merleau-Ponty (1962), in the area of perception, and Schutz and Luckmann (1974) in the social arena. Merleau-Ponty shows by close analysis of our naive experience of our bodies, 
of perception and movement, and of our lived experience of time and space, how consciousness patterns a world around it and gives our lives a lived expressive unity. Gibson (1986) also, although appearing to contradict Maturana and Varela with his theory of "information pickup," offers a compatible approach to theirs which develops an ontology of the environment in terms of substances, surfaces, textures, illumination, and other animals, rather than using more abstract qualities such as form or color. Schutz and Luckmann develop a detailed account of the structures of the "life-world" in terms of its spatial, social, and temporal arrangements; what is more, they show how the living subject experiences the world in the course of life.

In the field of information systems, a phenomenological view has been put forward by Boland (1987), who suggests that we should recast the concept of information so that, instead of referring to something externally present and independently meaningful, it denotes instead a process of inward formation by which people assimilate what happens to them and integrate it into their lived experience.

These works give us the starting point for developing an ontological perspective in IS on an autopoietic foundation. This would lead to a more radical reformulation of the discipline and practice of information systems than the other two approaches discussed above. We would have to develop a much richer approach to the design of IS than afforded by our current abstract conceptual tool set. The challenge is to build systems that support, reflect, and project their users' inward assimilation of their lived experiences in a world constituted culturally, geographically, and historically. The technology may already be available, but the ontologically oriented development framework remains to be created.

\section{CONCLUSION}

It would be a legitimate conclusion to say that the whole discipline of IS is so undermined by the theory of autopoiesis that it ought to be dismantled. This is unlikely to happen, if only because of the practical utility of the discipline. At the very least, the critique and rejection of the idea of information in the theory of autopoiesis need to be addressed. The consequences of organizational closure and the rejection of instructive interactions also need to be studied and understood in IS. The application of metaphors from autopoiesis in IS may be useful, but is no real substitute for a serious consideration of the theory. A Luhmannian elaboration of autopoietic theory looks feasible and would advance the theoretical debate (and probably practical development) in IS. Development in IS of the ontological side of autopoiesis would be more difficult, but would in my view be truer to the direction of the original theory, and would be likely to lead to a reconceptualization of information which was more closely tuned to 
users' experiences. I have suggested that this work might be started from a phenomenological perspective.

\section{REFERENCES}

Beeson, I., and Davis, C. "Emergence and Accomplishment in Organizational Change," Journal of Organizational Change Management (13:2), 2000, pp. 178-189.

Boland, R. J. Jr. "The In-formation of Information," Chapter 14 in Critical Issues in Information Systems Research, R. J. Boland Jr. and R. A. Hirschheim (eds.), Chichester, England: John Wiley \& Sons, 1987.

Capra, F. The Web of Life, London: Harper-Collins, 1997.

Dell, P. F. "Understanding Bateson and Maturana: Toward a Biological Foundation for the Social Sciences," Journal of Marital and Family Therapy (11:1), 1985, pp. 1-20.

Dietz, J. L. G., and Widdershoven, G. A. M. "Speech Acts or Communicative Action?" in Proceedings of the Second European Conference on Computer-Supported Cooperative Work, L. Bannon, M. Robinson, and K. Schmidt (eds.), Amsterdam, 1991, pp. 235-248.

Gibson, J. J. The Ecological Approach to Visual Perception, Hillsdale, NJ: Lawrence Erlbaum Associates, 1986.

Grint, K., and Woolgar, S. The Machine at Work, Cambridge, MA: Polity Press, 1997.

Kickert, W. J. M. "Autopoiesis and the Science of (Public) Administration: Essence, Sense and Nonsense," Organizational Studies (14:2), 1993, pp. 261-278.

Luhmann, N. Ecological Communication, Cambridge, MA: Polity Press, 1989.

Luhmann, N. Social Systems, Stanford, CA: Stanford University Press, 1995.

Maturana, H. R., and Varela, F. J. Autopoiesis and Cognition: The Realization of the Living, Dordrecht, The Netherlands: D. Reidel, 1980.

Maturana, H. R., and Varela, F. J. The Tree of Knowledge, Boston: Sahmbhala, 1987.

McLoughlin, I. Creative Technological Change, London: Routledge, 1999.

Merleau-Ponty, M. Phenomenology of Perception, London: Routledge \& Kegan Paul, 1962.

Mingers, J. "The Cognitive Theories of Maturana and Varela," Systems Practice (4:4), 1991, pp. 319-338.

Mingers, J. Self-Producing Systems, New York: Plenum Press, 1995.

Morgan, G. Images of Organization (Chapter 8), Thousand Oaks, CA: Sage Publications, 1997. Schutz, A., and Luckmann, T. The Structures of the Life-World, London: Heinemann, 1974. Von Krogh, G., and Roos, J. (eds.). Managing Knowledge, London: Sage Publications, 1996. Winograd, T., and Flores, F. Understanding Computers and Cognition, Norwood, NJ: Ablex, 1986.

\section{About the Author}

Ian Beeson lectures in Information Systems at the University of the West of England in Bristol, UK. He is a member of the Community Information Systems Centre there and has a particular research interest in the potential for creative and empowering uses of information and communication technologies in community settings. He can be reached by e-mail at ian.beeson@uwe.ac.uk. 